\title{
Filmer le travail : apports d'une pratique de cinéma documentaire en géographie
}

Filming the work: contribution of the documentary filmmaking to the geography

Marie Chenet

\section{OpenEdition}

Journals

Édition électronique

URL : http://journals.openedition.org/itti/392

DOI : 10.4000/itti.392

Éditeur

Université de Poitiers

\section{Référence électronique}

Marie Chenet, «Filmer le travail : apports d'une pratique de cinéma documentaire en géographie », Images du travail, travail des images [En ligne], 8 | 2020, mis en ligne le 01 février 2020, consulté le 14 avril 2021. URL : http://journals.openedition.org/itti/392 ; DOI : https://doi.org/10.4000/itti.392

Ce document a été généré automatiquement le 14 avril 2021

Images du travail, travail des images 


\section{Filmer le travail : apports d'une pratique de cinéma documentaire en géographie}

Filming the work: contribution of the documentary filmmaking to the

geography

Marie Chenet

L'auteur remercie les trois relecteurs, Baptiste Buob, Jean-Paul Géhin et un.e personne anonyme, qui ont pris le temps d'évaluer ce texte et dont les commentaires ont considérablement amélioré cet article.

\section{Introduction}

1 Les films réalisés par des chercheurs en géographie commencent timidement à trouver leur place dans la géographie française. Si les recherches doctorales recourant au film se multiplient, la communauté des géographes revendiquant la réalisation filmique comme outil de recherche à part entière est encore restreinte à une quinzaine d'individus en France (pour un aperçu, voir la liste des intervenants au colloque «Le film dans la pratique de la géographie $»^{1}$ qui s'est tenu à Bordeaux en mars 2018). Parmi eux, la majorité utilise l'outil filmique à des fins de médiation au service d'une démarche participative de la recherche. Plus rares sont ceux qui revendiquent la réalisation de films documentaires comme fin en soi, afin de produire et de formaliser de façon singulière des savoirs géographiques avec les outils du cinéma (Raoulx, 2018). Cette approche méthodologique, est anciennement commentée dans le champ des sciences sociales (Naville, 1966; Terrenoire, 1985; Rose, 2001) et encore plus spécifiquement par les chercheurs de l'anthropologie visuelle ou filmique (de France, 1989 ; Ruby, 2000 ; Buob, 2009 ; Colleyn, 2012) et par certains sociologues (Sebag, 2012).

2 C'est dans ce courant que je m'inscris en réalisant des films documentaires au sein de mes recherches en géographie. L'objectif est de produire des œuvres inédites, affichant 
une démarche d'auteur, pour incarner et documenter des processus territoriaux. Depuis 2009, j'ai ainsi réalisé ou coréalisé six films documentaires de durées variables (de 4 à 42 minutes). Ces films ont été produits dans des cadres divers: projets personnels et spontanés ou projets intégrés à des programmes de recherche. De ce corpus se dégage la thématique récurrente du lien qui unit les individus à leur milieu naturel. De ces six films, trois d'entre eux mettent en scènes des individus au travail. À partir de cette expérience, je propose de m'interroger sur la façon dont filmer des travailleurs et des territoires peut contribuer à enrichir les savoirs géographiques, mais aussi à enrichir la pratique de la géographie elle-même. L'objectif est de contribuer à montrer l'intérêt de produire des œuvres de cinéma documentaire dans le champ de la recherche en sciences humaines, et plus spécifiquement en géographie.

\section{Filmer le travail en tant que géographe-cinéaste}

\section{Cinéma documentaire et géographie : une démarche encore marginale en France}

3 Les géographes anglo-saxons ont bien formalisé l'usage de la vidéo au sein de la discipline, mettant l'accent principalement sur l'apport de la pratique filmique aux méthodes participatives (Kindon, 2003; Dwyer et Davies, 2010; Garett, 2011, 2013, vol. 48/4 de la revue Area, 2016), mais aussi sur l'intérêt de produire des œuvres documentaires comme production scientifique à part entière (Spinney, 2009 ; Simpson, 2011 ; Jacobs, 2015 ; Baptiste, 2016). Par son aspect multi-sensoriel et multi-modal, les auteurs britanniques ont insisté notamment sur le fait que le film est un outil de recherche approprié pour rendre compte de l'expérience de l'espace vécu (Jewitt 2009; Pink 2011, Marion and Crowder 2013).

4 En France, des géographes ont réalisé des films documentaires dès les années 1960 au sein du centre Audiovisuel de l'ENS Saint-Cloud à des fins pédagogiques (Chenet et al., 2011). Mais ce n'est qu'à partir des années 1990 que des chercheurs revendiquent l'intérêt de réaliser des films afin de formaliser et valoriser autrement les savoirs géographiques (entre autres Browaeys, 1999; Raoulx, 2009 ; Buire, 2011; Collignon, 2012). L'affirmation de l'adoption du cinéma documentaire comme outil au sein de la discipline, afin de produire une formalisation des connaissances singulière, est plus récente. Par cinéma documentaire, nous désignons le genre cinématographique qui utilise des éléments du réel et qui se distingue de la production audiovisuelle classique par la revendication d'un point de vue d'auteur afin de produire de nouvelles relations entre des éléments captés par une caméra (Niney, 2009). En ce sens, seule une poignée de géographes revendique une démarche d'auteur dans la réalisation de films géographiques, au carrefour entre recherche et cinéma documentaire (Ernwein, 2015 ; Chenet, 2016; Raoulx, 2018; Chouraqui, 2018), c'est dans cette veine que nous inscrivons notre travail filmique.

\section{Filmer le travail en tant que géographe : trois expériences de film}

$5 \quad$ Peu de films de géographes ont eu pour objet le travail (citons tout de même les travaux de Xavier Browaeys, Benoit Raoulx et Marion Ernwein). J’ai pour ma part réalisé trois 
films documentaires qui montrent les liens qui unissent des travailleurs et un territoire.

Image 1

\section{Entre chiens et loups}

un film de Marie Chenet

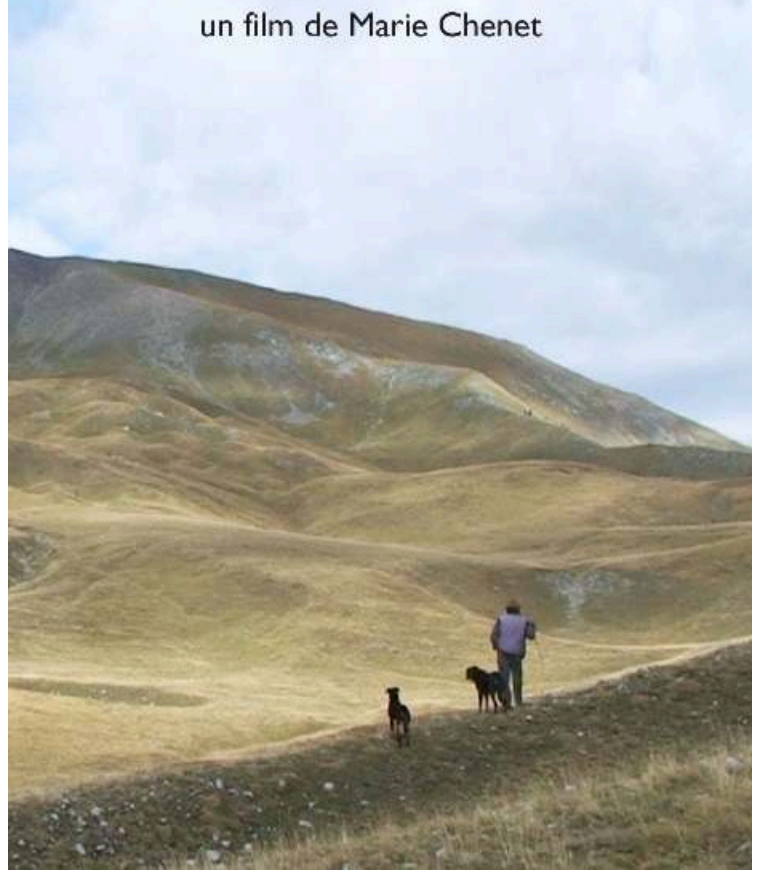

Mon premier film documentaire, Entre Chiens et Loups², réalisé en 2010.

6 Tourné en septembre 2009 dans le Parc national du Mercantour, ce film traite de la difficile cohabitation entre l'activité pastorale et la présence du loup dans les Alpes françaises depuis son retour dans les années 1990. À travers le portrait de Thierry Giordan, éleveur-berger ovin, le film se veut une immersion dans le quotidien d'un travailleur de la montagne qui doit faire face à l'intrusion d'une nouvelle espèce sur son espace de travail, mais aussi à des changements d'usage et de conditions de vie dans les territoires montagnards. J'ai décidé de filmer Thierry Giordan au cours d'un été alors que je travaillais comme aide-bergère. Le projet de film venait donc s'associer à cette mission première consistant à «travailler au troupeau». Le film qui en a résulté correspond assez bien à ce que j'avais écrit avant le tournage : découpé en trois parties, il présente d'abord le berger comme le gardien solitaire de sa montagne, maître de son territoire, puis la lutte qu'il doit mener contre un ennemi bien présent mais toujours invisible, et enfin la reconnaissance de la défaite dans une guerre qui oppose le berger au loup. Le film est également l'occasion d'évoquer les tensions entre un monde agricole traditionnel et un monde plus moderne et urbain. 


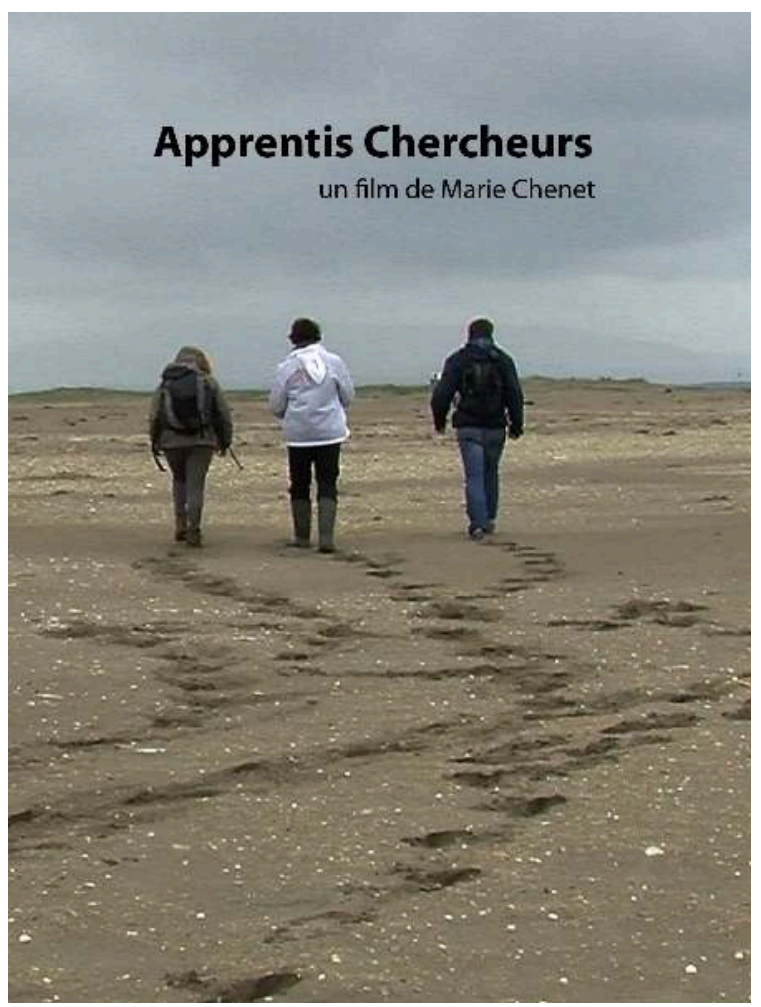

Apprentis Chercheurs ${ }^{3}$ est le deuxième film documentaire que j'ai réalisé, en 2013

7 Il suit l'apprentissage d'étudiants de Licence en Géographie au cours d'un stage de terrain de géomorphologie en Irlande. Les questionnements principaux du film sont ceux de la pédagogie, de la transmission des connaissances sur le terrain, mais aussi de l'apprentissage de la recherche scientifique. Il est construit autour de trois figures de l'apprentissage : un professeur des universités en fin de carrière (Charles Le Cœur), des maîtres de conférences fraîchement recrutés (au nombre de quatre, dont moi-même) et des étudiants (ici un groupe d'une vingtaine d'individus). J'occupais dans ce film une place singulière: je filmais à la fois mes étudiants, mes collègues, mais aussi le professeur qui m'avait formé et encadré lors de ma thèse de doctorat. Le film suit un déroulé chronologique des premières leçons professorales in situ à l'appropriation par les étudiants du décryptage des paysages. La forme autobiographique s'est imposée au montage : devant la difficulté à faire comprendre, par les images et les interactions entre les personnages, mon statut particulier d'ancienne étudiante et d'enseignante, ainsi que mes questionnements sur la transmission des savoirs, j'ai adopté un récit à la première personne du singulier. 


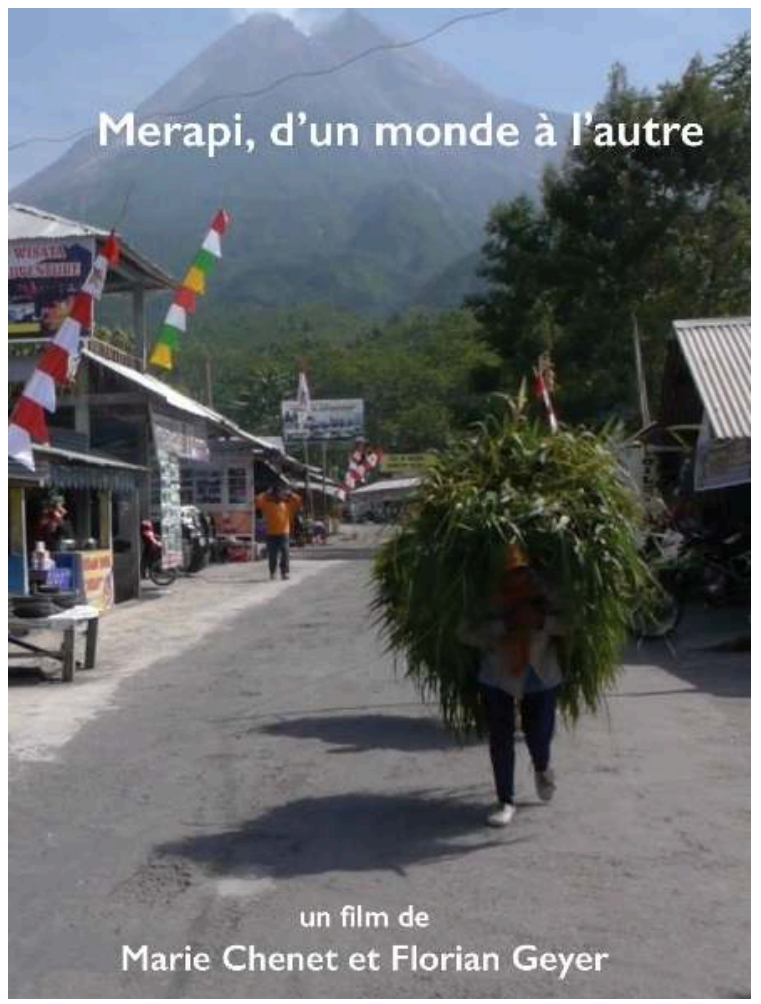

Le troisième film dans lequel j'ai filmé des individus en situation de travail a été coréalisé avec Florian Geyer en 2016 et s'intitule Merapi, d'un monde à l'autre 4 .

8 Produit dans le cadre du projet de recherche Sedimer (2012-2014, financement Fondation Axa), il traite de la façon dont le territoire dévasté par l'éruption du volcan Merapi en 2010 (Java, Indonésie) est réinvesti par la population locale, tant sur le plan économique que socioculturel, quatre ans après la catastrophe. On y voit notamment le volcan comme une ressource économique puisque l'éruption a permis l'essor d'une activité touristique tous azimuts, mais aussi une intensification de l'exploitation des sables volcaniques qui a attiré de nombreux travailleurs dans la région. Nous montrons également comment l'éruption s'est soldée par une réorganisation des structures sociales (passage d'un habitat dispersé à un habitat regroupé, modification des pratiques agricoles) et l'abandon d'un islam empreint de syncrétisme au profit d'un islam «arabique ». Le film est construit comme un cheminement allant des sites détruits par l'éruption aux nouveaux lieux de vie de la population. Dans chaque lieu, des acteurs locaux racontent la façon dont ils ont vécu et accompagné ce bouleversement territorial.

9 Ces trois films, comme les autres films que nous avons réalisés, ont été projetés dans des cadres divers. Ils ont pu faire l'objet de programmations dans des colloques, des séminaires ou des festivals de géographie (entre autres FIG, Géocinéma) mais aussi des festivals plus spécifiquement de cinéma (entre autres Rencontres du cinéma Européen à Vannes, Festival international cinéma nouvelle génération à Lyon, Filmer le Travail à Poitiers). La multiplicité des projections nous a ainsi permis d'élargir notre réflexion de recherche en nous confrontant, par le débat, à des appréciations de non-géographes, mais aussi aux retours directs des personnes apparaissant dans nos films. 


\section{Filmer les travailleurs et leurs territoires : contributions à différents champs de la géographie}

10 Les géographes dont les recherches ont pour objet le travail, sont généralement rattachés au courant de la géographie sociale, s'intéressant au travail dans sa relation à la structuration sociale de l'espace. Dans le sillage des recherches pionnières de Renée Rochefort sur les travailleurs siciliens (Rochefort 1961, 1963a, 1963b ; Aldhuy, 2006) et des écrits d'Henri Lefebvre (1974), cette géographie sociale s'attache à l'analyse des relations entre rapports sociaux et spatiaux et conçoit l'espace comme le produit de dynamiques sociales. Il s'agit donc d'envisager l'espace à l'échelle de l'individu et de procéder à une triple analyse des formes de l'espace, de ses pratiques et de ses représentations (Di Méo, 2016). C'est précisément cette approche que revendiquent Benoît Raoulx (2009) et Marion Ernwein (2015), rares géographes à avoir filmé des travailleurs. Pour ma part, je ne m'inscris pas dans ce courant et suggère que ma façon de filmer des travailleurs contribuent à d'autres champs de la géographie.

\section{Filmer le travail, contribution à une géographie environnementale incarnée}

11 Géomorphologue de formation, j'ai développé des recherches sur la réponse des milieux naturels aux changements environnementaux, essentiellement en milieu montagnard. En parallèle de mes travaux de géomorphologie, j'ai recours au cinéma documentaire pour documenter la relation qu'entretiennent les individus avec le milieu naturel. Le cinéma présente l'intérêt de pouvoir travailler à l'échelle de l'individu constituant ainsi un puissant outil d'incarnation des processus territoriaux. Dans trois de mes films, je documente l'expérience de travailleurs au sein de milieux naturels qui ont connu des changements environnementaux majeurs (réapparition du loup dans les Alpes françaises, éruption volcanique à Java) ou qui servent de lieux d'apprentissage (expérience pédagogique en Irlande). Dans les trois cas, le milieu naturel est tour à tour un espace que l'on exploite ou que l'on subit au grès des aléas naturels ou du degré de maîtrise de certains outils.

Dans Entre chiens et loups, c'est le retour dans les Alpes françaises au début des années $1990 \mathrm{du}$ loup gris (Canis Lupus) qui engendre des bouleversements dans les pratiques pastorales traditionnelles ovines et caprines. Le film montre la façon dont un berger se voit contraint de modifier ses pratiques de travail pour faire face à l'arrivée du prédateur. Malgré ces efforts, le loup continue à prélever des bêtes au troupeau, mettant en péril la pérennité de l'activité pastorale. Le film est construit de façon à montrer comment, dans une "guerre » entre l'homme et la nature, le berger passe du statut de gardien de la montagne et détenteur de savoirs séculaires à un statut de perdant, dont la seule issue serait la retraite. Au-delà de ce conflit, il laisse progressivement penser que la menace n'est peut-être pas la présence du loup en ellemême, mais les décisions d'un pouvoir lointain (en l'occurrence celui de l'État français et de l'Union Européenne) déconnecté des réalités locales. Pour soutenir cette idée, le film insiste dans sa deuxième moitié sur le sentiment d'abandon et d'isolement du berger qui se retrouve acculé dans sa cabane par le brouillard et le mauvais temps.

Dans Merapi, d'un monde à l'autre, c'est aussi un phénomène naturel qui modifie les pratiques de travail. L'éruption du volcan Merapi en 2010 s'est soldée par la destruction 
de trois villages, la mort de 380 personnes et l'évacuation de 400000 personnes. Le film montre comment, quatre ans après l'éruption, le temps de la catastrophe a laissé la place au temps de l'exploitation des ressources du volcan. Filmée du point de vue des travailleurs, l'éruption est vécue comme source de profit à travers l'exploitation touristique et minière. Le tourisme est fondé à la fois sur l'attraction de la figure mythique du "gardien du volcan", Mbah Marijan, mort pendant l'éruption (de nombreux visiteurs viennent se recueillir sur les ruines de sa maison) et sur l'exploitation des paysages dévastés par l'éruption, offrant un terrain de choix pour l'organisation de tours en jeep à travers les cendres et les ruines des villages. Quant à l'activité minière, elle consiste à extraire les formidables quantités de sables et de pierres volcaniques fournies par l'éruption et qui sont utilisées dans le secteur de la construction. La parole des travailleurs révèle les stratégies individuelles et collectives pour exploiter le territoire, mais aussi les conflits d'usage entre les différentes activités. L'extraction de sable, dont les camions endommagent fortement les routes, pénalise ainsi l'activité touristique mais aussi agricole, puisque les agriculteurs aimeraient pouvoir de nouveau exploiter les terres pour le fourrage.

Le film Apprentis Chercheurs a un statut particulier, puisqu'il ne traite pas de l'impact de changements environnementaux, mais d'une expérience pédagogique au sein d'un milieu naturel. Il montre comment l'acquisition des connaissances géomorphologiques relève d'un cheminement à travers les paysages, cheminement qui doit aboutir à l'autonomie des étudiants dans la compréhension du fonctionnement des milieux naturels. Ce cheminement passe par la confrontation aux éléments naturels (escalader des parois, goûter l'eau), l'acquisition d'outils (le dessin, la mesure), la maîtrise de vocabulaire, le dépaysement, l'égarement, mais aussi le plaisir du questionnement et l'appréciation de l'esthétisme des paysages.

Le cinéma documentaire permet de documenter des expériences individuelles en adoptant un point de vue singularisé. Loin de viser à l'exhaustivité, le film se veut une incarnation de processus territoriaux à l'échelle de l'individu. Dans nos recherches, filmer le travail donne à voir et à entendre le point de vue singulier de travailleurs exploitant des milieux naturels et révèle un rapport ambivalent à la Nature, successivement menaçante et bienfaitrice. Pour profiter de ces espaces, il faut bien souvent accepter de lui payer un tribut, mais aussi composer avec des politiques de gestion environnementales qui se placent rarement à l'échelle individuelle. Pour faire comprendre et accepter cette ambivalence au spectateur qui a parfois un point de vue divergent sur l'exploitation des milieux naturels, le géographe-cinéaste peut jouer sur l'empathie qu'il développe avec ses personnages, puisque le cinéma agit par ce que J.-P. Colleyn (1993) appelle une « familiarisation stimulante».

\section{Filmer le travail, contribution à la géographie des corps}

Filmer le travail permet également de donner à voir des corps en action. Au sein du courant de la géographie culturelle, les géographes français ne se sont que récemment intéressé à la géographie des corps (Volvey, 2000, 2014; Hancock, 2011; Fournand 2008 ; Barthe-Deloizy, 2011 ; Séchet, 2012 ; Jaurand, 2015 ; Staszak et al., 2018). Centrée sur l'étude de milieux urbains, la géographie des corps s'interroge sur les rapports qu'entretiennent mutuellement l'espace et le corps: comment les relations spatiales s'articulent pour composer le corps et comment le lieu est composé à travers le corps? Des géographes britanniques ont pu avoir recours à la vidéo pour traiter de ces 
questions de corporéité (Laurier 2001, 2005 ; Laurier et al., 2006 ; Simpson, 2011), mais ils ont utilisé la vidéo comme un outil d'enregistrement de pratiques corporelles et ne s'inscrivent donc pas dans une démarche documentaire. De plus, toutes ces études traitent de pratiques en milieu urbain, ce qui rend leurs résultats difficilement comparables avec les nôtres.

Pour reprendre les mots de Francine Barthe-Deloizy (2011), mes films traitant du travail témoignent de "l'expérience spatiale des corps " puisqu'ils donnent à voir un corps à corps entre le milieu naturel et les travailleurs. Le film permet en effet de mettre en valeur la corporéité des individus en faisant entendre leur respiration, leur timbre de voix, les sons ambiants, en rendant compte de leur champ visuel et en restituant la temporalité des trajectoires qu'ils effectuent dans les paysages. Le milieu est également révélé par ses attributs physiques élémentaires: les conditions climatiques et atmosphériques, les aspérités topographiques sont autant d'éléments qui interagissent avec les organismes humains.

Dans Entre chiens et loups, la rudesse du territoire (versants escarpés, froid, brume, pluie, cris stridents de marmottes ou de vautours) participe petit à petit de l'usure du corps du berger et rend d'autant plus pénible le combat qu'il faut mener contre le loup. J'ai ainsi construit le film sur la mise en valeur de plus en plus prégnante de la pénibilité du travail. Pour ce faire, j'ai mis l'accent sur les attitudes du corps du travailleur et sa place dans les paysages (Image 4). En début de film, j'ai laissé la part belle aux plans larges de paysages montagnards majestueux, dans lesquels le berger apparaît à la fois comme un élément naturel parmi d'autres, mais également le maître des lieux (Image 4a). J'ai également mis en avant la maîtrise des gestes de travail (travail avec les chiens de conduite, soins aux bêtes, etc.) et l'aisance avec laquelle il évolue entouré de ses bêtes (Image $4 \mathrm{~b}$ ). Parallèlement, il est interviewé en extérieur, sous le soleil, de plainpied (Image 4c). Au fur et à mesure du film, le berger, qui se révèle acculé par le loup et par les autorités institutionnelles, se plaint des intempéries et de la pénibilité du travail. Le souffle de sa respiration est de plus en plus audible, les versants semblent plus difficiles à gravir (Image 4d). Son ras-le-bol de la situation s'exprime lors d'entretiens filmés dans la cabane, le soir, autour d'une table, ses épaules sont alors voûtées et sa chemise déchirée (Image 4e). La caméra elle-même semble se rapprocher de ce corps malmené. 
Image 4 : «Évolution de la posture du corps du berger et de son rapport au paysage » dans le film Entre chiens et loups (Chenet, 2010)

Image 4a. « Le berger à la garde du troupeau »

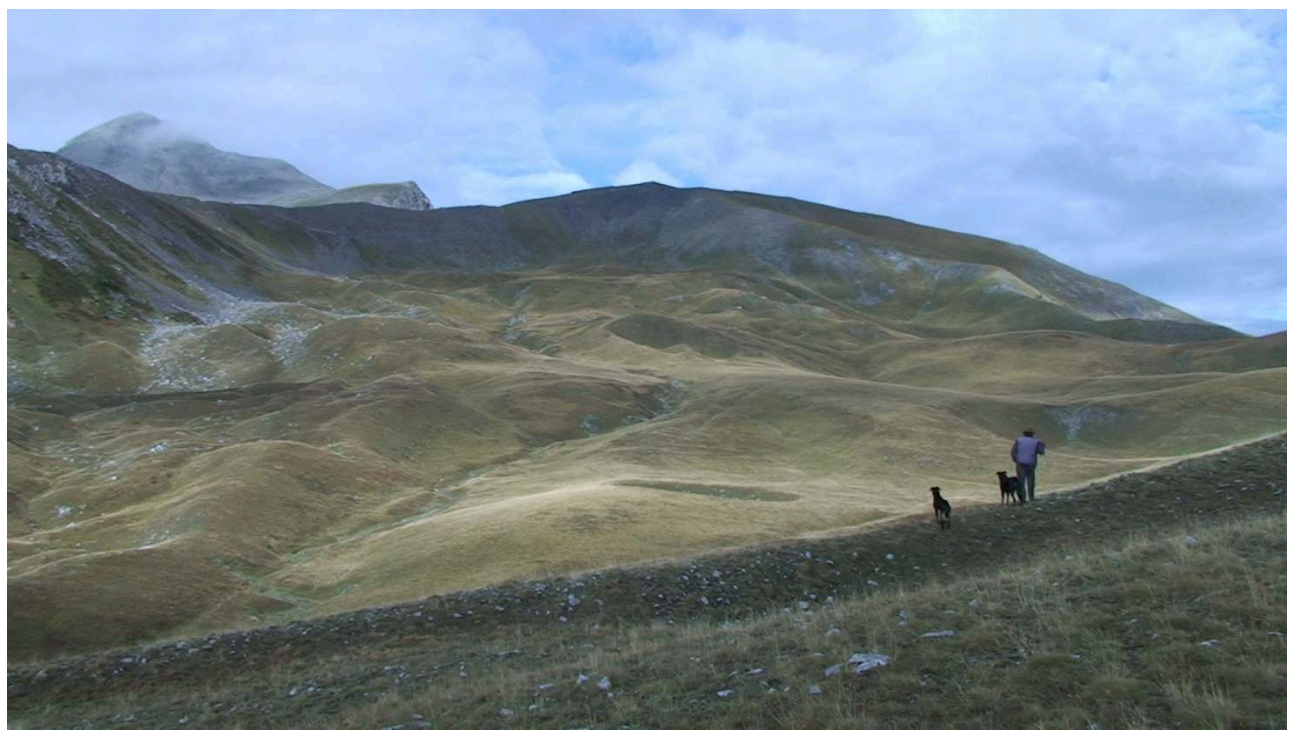

Image 4b. « Le soir, rentrée des bêtes au parc »

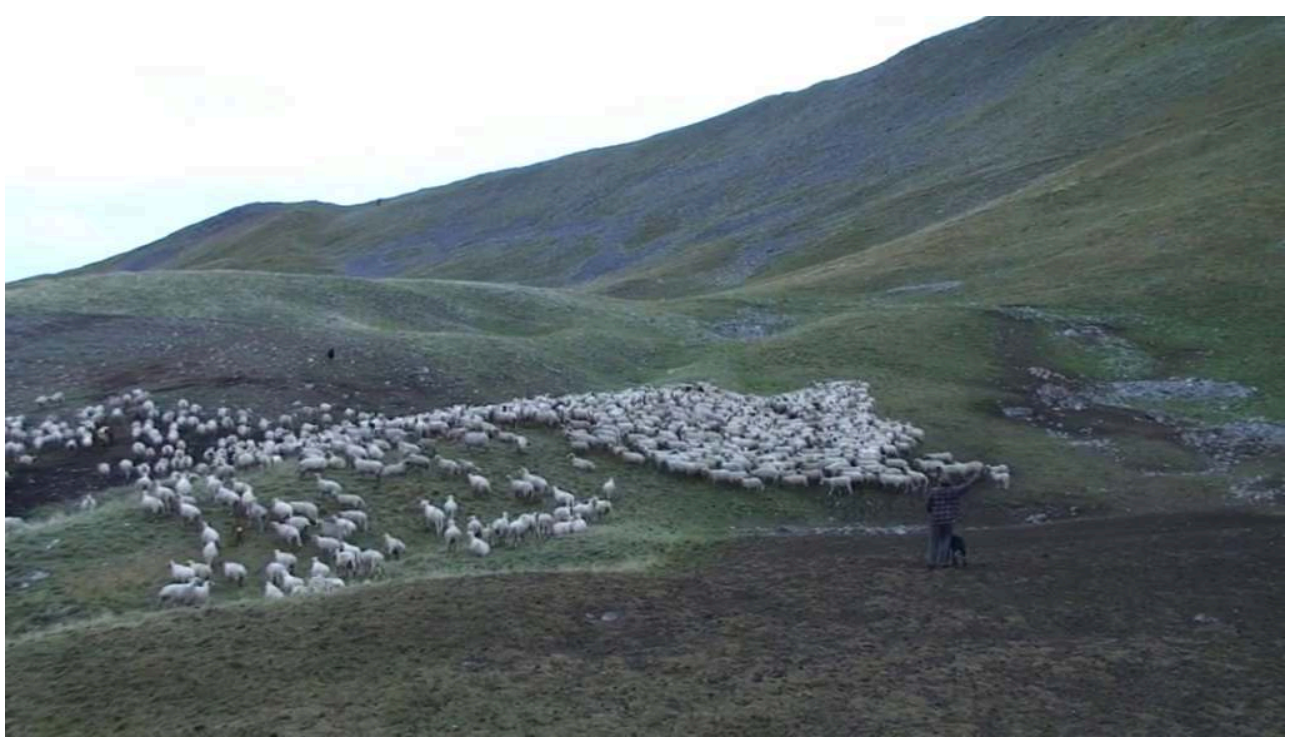


Image 4c. « Interview en extérieur, de plain-pied»

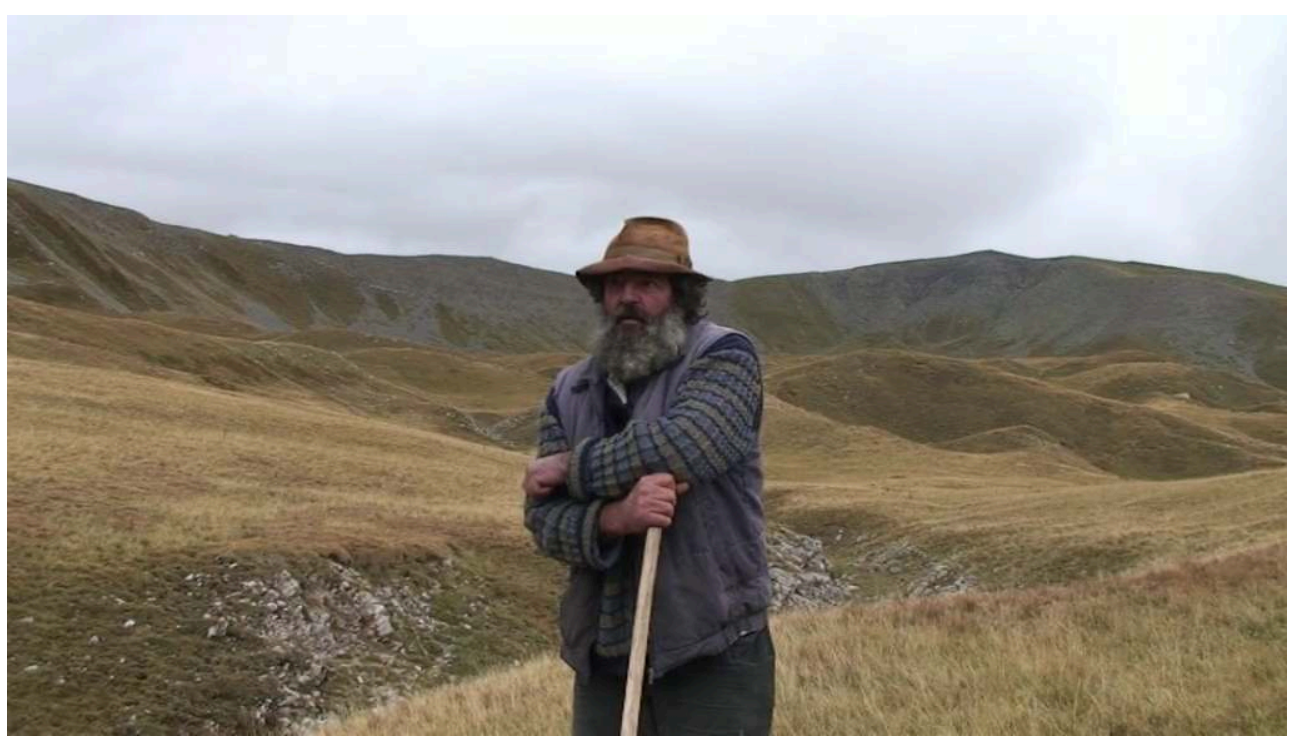

Image 4d. « Retour de garde, sous la pluie »

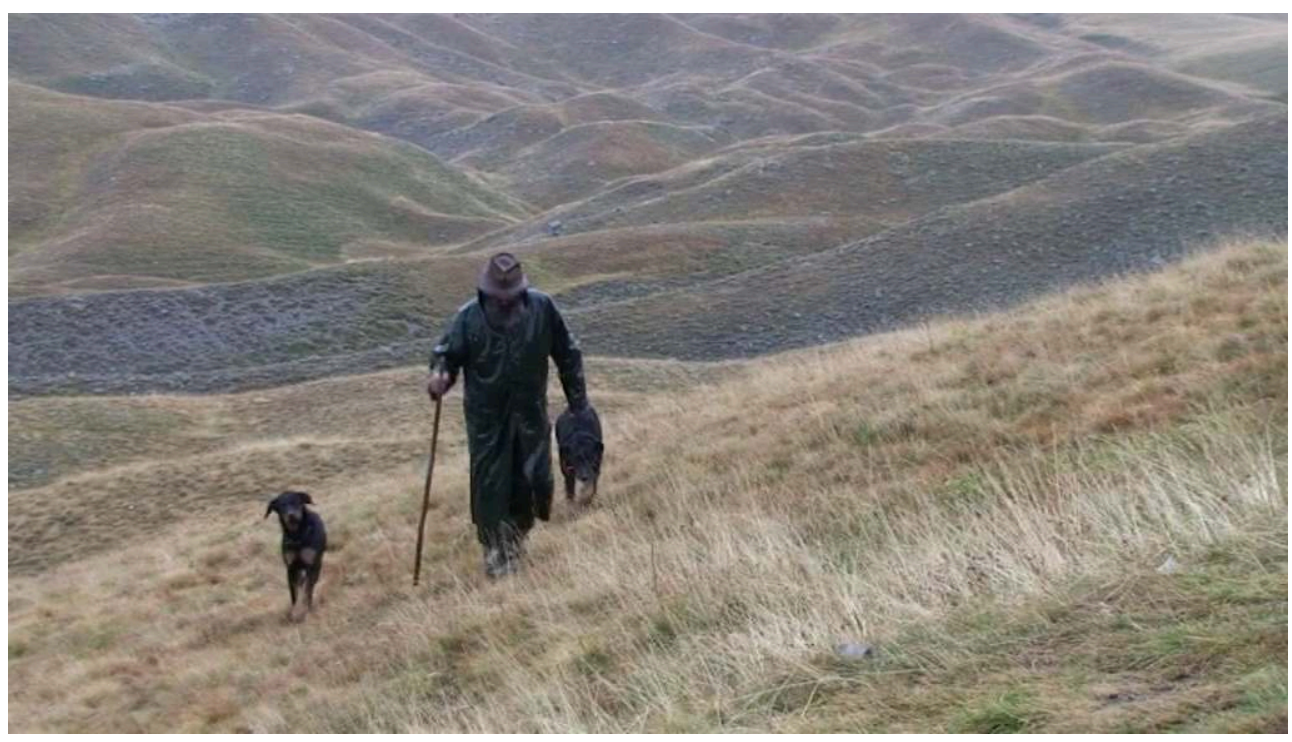


Image 4e. «Interview le soir, dans la cabane »

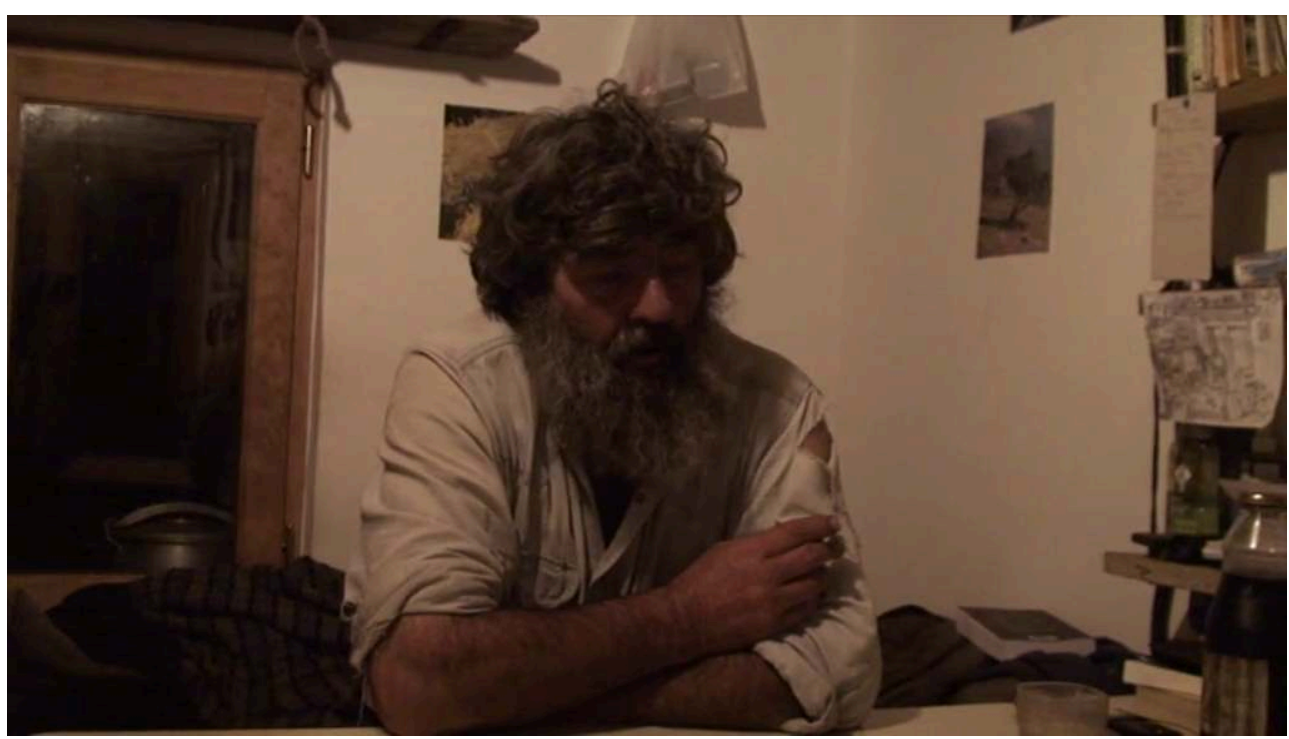
qu'elle engendre, la traversée en jeep des paysages dévasté, saturés de poussière des cendres volcaniques et du son des moteurs et des haut-parleurs, est vécue comme une aventure excitante par les touristes. L'épreuve des corps participe de l'expérience touristique et est mise en valeur par les nombreux clichés pris tout au long de la visite. Les poses se font à la fois dominatrices et conquérantes. Dans ces paysages, les travailleurs se font plus discrets voir invisibles, ils s'effacent face au flux touristique (Vidéo 1). Les agriculteurs apparaissent en arrière-plan, ployant sous des tas de fourrage charriés sur le dos. Les travailleurs associés au tourisme (gardiens de site, vendeurs, conducteurs de jeeps) interviennent eux rapidement pour ramasser les déchets ou récupérer l'argent des touristes. Le film met également en avant les temps d'attente entre deux passages de clients. Le corps est alors au repos ou aux aguets, révélant des corps au diapason avec les flux touristiques. Du côté de l'extraction minière, les corps travaillant ont un autre statut puisqu'ils constituent un spectacle pour les touristes. Ces forçats qui cassent des pierres à la masse, charrient de grands volumes de sables au moyen de tractopelles tonitruants ou font s'effondrer un pan de montagne sous un soleil rutilant semblent participer à l'affrontement qui oppose les hommes au volcan (Vidéo 2). Si le volcan a pris des vies lors de l'éruption, il est maintenant dominé par cette force de travail.

Ce média ne peut être affiché ici. Veuillez vous reporter à l'édition en ligne http:// journals.openedition.org/itti/392

Ce média ne peut être affiché ici. Veuillez vous reporter à l'édition en ligne http:// 
naturels développent bien souvent un rapport émotionnel et sentimental avec leur territoire. La géographie s'est peu intéressée à ce que Smith et Anderson (2001) nomme les sentiments "souterrains ", désintérêt qui peut être relié à la difficulté de mettre en mots ces sentiments (Davidson et al., 2005). Le film apparaît comme un outil approprié pour pallier à cette difficulté (Jacobs, 2015). J. Lorimer (2010) a ainsi montré comment l'analyse d'images filmiques pouvaient révéler des liens émotionnels qui unissent des éléments humains et non-humains (dans son cas, des éléphants). Nous allons plus loin en suggérant que le recours à des éléments cinématographiques qui relèvent du sensoriel (ambiances sonores, choix de cadrage, esthétisme des cadres) insérés au sein d'un montage pensé par le cinéaste-géographe permet de restituer des émotions qu'il a pu lui-même capté sur le terrain et qui sont difficilement dicibles.

Entre Chiens et loups montre par exemple qu'au-delà de la relation de travail qui existe entre le triangle bergers-chiens de conduite-troupeau, il existe également des liens sensibles indispensables à la vie dans ce milieu vide d'humains. J'ai accordé une 
attention particulière aux liens affectifs qui unissent Thierry à ses bêtes, notamment à travers la séquence de soins où en plans rapprochés, on le voit enlacer une brebis et lui murmurer à l'oreille des paroles réconfortantes (Vidéo 4). Ce lien est également souligné avec les chiens qui collent aux semelles de leur maître tout au long du film. L'apparition du loup peut être vu comme une menace pour cet équilibre affectif. La façon dont j'ai filmé les paysages majestueux du Mercantour, en plan large et éclairé par des lumières qui les magnifient, participent également de la transmission du lien indicible qui unit Thierry à sa pâture. Dans le film sur le Merapi, l'émotion surgit dans les silences des travailleurs qui observent de loin le balai des touristes utilisant comme terrain de jeu leur territoire dévasté. Nous avons joué sur ce décalage notamment avec des éléments sonores : la joie des touristes est soulignée par le vacarme sonore des hauts-parleurs, du vrombissement des moteurs et des rires, tandis que l'évocation de l'éruption volcanique et des pertes associées se fait dans une atmosphère silencieuse. Cette ambivalence rythme le film, alternant séquences joyeuses et séquences plus lourdes. Dans Apprentis Chercheurs, l'émotion est présentée comme une condition sine qua non à la réussite du travail. L'appréciation esthétique des paysages est notamment soulignée comme moteur d'apprentissage. On y voit ainsi une scène où deux enseignants contemplent le paysage en silence, entrecoupé par quelques échanges d'ordre technique mais aussi esthétique. Cette idée sera reprise plus tard où des étudiantes invitent d'autres à les rejoindre en haut d'un versant pour profiter de la vue. La joie est également présentée comme un moteur d'apprentissage. Des scènes montrant le plaisir des enseignants à se poser des questions d'ordre scientifique sur un ton à la fois léger mais sérieux sont mises en parallèle avec la scène finale où les étudiantes s'adonnent à la chanson pour relater leur expérience de recherche.

Ce média ne peut être affiché ici. Veuillez vous reporter à l'édition en ligne http:// journals.openedition.org/itti/392

Les films révèlent donc que les travailleurs développent avec leurs milieux des liens émotionnels souvent indicibles, mais indissociables de leur pratique de travail. Ces liens apparaissent comme une condition essentielle à l'acceptabilité de la rudesse des conditions de travail et de l'exposition des corps et à la pérennité de l'activité. Dans ce contexte, la question du travail et l'outil film semblent être particulièrement pertinents pour appuyer l'intérêt d'une géographie des émotions telle qu'elle a été formalisée par quelques auteurs français (Bochet et Racine, 2002 ; Guinard, 2016 ; Guinard et Tradjnek, 2016).

\section{Une géographe et une caméra : un autre rapport à la recherche et à l'espace}

Cette partie présente la façon dont le recours à la caméra modifie les questionnements mais aussi les conditions de recherche du géographe. L'adoption de la forme cinématographique impose en effet au chercheur de se poser des questions concernant son point de vue, les procédés filmiques ou l'échelle d'observation. Mais le recours à une caméra entraîne d'autres effets : il peut être un révélateur du positionnement du chercheur sur son terrain, voire un moyen de négocier sa place dans l'espace. Cette 
présence génère un autre type d'espace, une scène d'exposition avec laquelle le géographe-cinéaste doit composer.

\section{La caméra comme révélateur de la position du chercheur sur le terrain}

31 La spécificité de la démarche documentaire est que le cinéaste-géographe doit se poser la question du point de vue adopté dans son film. Par point de vue, s'entend à la fois le propos développé par le chercheur, mais également la position de celui-ci sur le terrain et la vision qu'il va nous faire adopter pour fonder son argumentation. C'est donc une démarche qui répond bien aux exigences des sciences sociales qui privilégient un regard sur une réalité observée, s'interrogent sur la construction de cette réalité et explicite les conditions précises selon lesquelles le chercheur se livre à cet exercice. Le film documentaire permet de rendre explicites les conditions de production des connaissances, en intégrant les caractères personnels du chercheur-cinéaste révélés par son style et en donnant à percevoir les traces de la négociation de la relation chercheur - enquêté (Hémont et Patrascu, 2016). Par le film, le géographe-cinéaste peut ainsi suggérer sa position sur le terrain tant sur le plan spatial que statutaire par rapport à ses enquêtés et révéler son échelle d'observation. La multiplication récente d'écrits de géographes qui questionnent et critiquent la position et le rôle du chercheur sur son terrain (Volvey, 2003; Lévy et al., 2004; Calbérac, 2011; Buire, 2012; Verne, 2012) témoigne de cette préoccupation croissante, mais plus rares sont les géographes qui mentionnent la caméra comme outil de révélation du positionnement du géographe sur son terrain (Calbérac, 2010 ; Buire, 2011 ; Ernwein, 2015).

Dans les trois films que j'ai réalisés, cités ci-dessus, la question du point de vue a toujours été posée dès le début du processus d'écriture. Vais-je raconter mon récit du point de vue du chercheur qui mène son enquête ou vais-je privilégier le point de vue revendiqué d'un ou plusieurs acteurs du sujet? Pour le film Entre Chiens et Loups, je voulais résolument évoquer le problème de la présence du loup en France du point de vue d'un éleveur-berger, le film a donc été construit sous la forme d'un portrait présentant le quotidien du protagoniste, quotidien perturbé par l'intrusion d'un prédateur. Le berger est quasiment de tous les plans, le cheminement à travers sa pâture se fait toujours à ses côtés, caméra au poing, et les plans larges permettent de le replacer dans l'immensité des paysages pelés de haute montagne. La caméra se rapproche progressivement pour l'observer de plus près au travail, jusqu'à se retrouver enfermée dans la cabane, où Thierry semble acculé par le loup. J'ai fait le choix de laisser mes échanges et mes questions pour expliciter la nature des relations que j'ai eu avec mon personnage : celui d'une jeune citadine un peu ingénue qui vient partager le quotidien de son personnage et qui échange sur le problème du loup. Je fais donc de ma propre personne un personnage secondaire auquel le spectateur peut s'identifier.

Dans le film Apprentis Chercheurs, je savais dès le départ que ma fonction d'enseignante qui filme ses collègues et ses étudiants n'était pas anodine dans le propos tenu. Je me place donc en observatrice de mon professeur et des étudiants, comme un personnage intermédiaire entre ces deux générations. Mon professeur est ainsi souvent filmé de façon individuelle et en légère contre-plongée, alors que les étudiants sont toujours filmés en groupe. Avec mes autres collègues, j'ai laissé les adresses à la caméra, allant même jusqu'à laisser ma caméra tourner sur pied pour les rejoindre dans le cadre. 
N'étant pas persuadée que ces seuls procédés filmiques permettraient de comprendre mon statut particulier dans ce film, j'ai finalement adopté au montage la forme du récit autobiographique, avec l'utilisation d'une voix off dite à la première personne.

Pour le film Merapi, d'un monde à l'autre, c'est au moment des repérages et de la période d'enquête sur le terrain que nous avons décidé avec Florian Geyer de présenter le territoire et ses problématiques sous l'angle de la population locale, plus particulièrement celui des travailleurs. Nous avons privilégié l'observation du terrain depuis les échoppes qui jalonnent le volcan où nous avons noué le dialogue avec les locaux qui tiraient profit du volcan et c'est ce point de vue que nous avons voulu retranscrire dans notre film. Bien sûr, les touristes sont présents dans le film, mais comme toile de fond, observés avec distance par nous-mêmes et par les habitants du volcan, depuis des intérieurs, par des fenêtres, des portes. Nous avons tout de même éprouvé la nécessité de signifier nos origines et notre propre place sur le territoire : dès les premiers plans du film, Florian Geyer, alors qu'il en train de filmer sur pied, est interpellé par un touriste qui souhaite être pris en photo, il rentre alors dans le champ tout en lui répondant en indonésien (Vidéo 5).

Ce média ne peut être affiché ici. Veuillez vous reporter à l'édition en ligne http://

Ces questionnements de positionnement concernant la distance et le rapport au terrain sont tout à fait inhérents à la pratique du cinéma. Comme le dit Serge Daney dans ses derniers entretiens, pratiquer le cinéma, c'est "trouver sa bonne distance au monde $\aleph^{5}$.Le recours au film implique donc pour le chercheur de réfléchir aux procédés cinématographiques et aux modes de narration qui permettent de révéler sa position sur le terrain. Cette approche inscrit ainsi la démarche du géographe-cinéaste dans les préoccupations de certains courants de la géographie sociale et culturelle qui préconisent une meilleure contextualisation et une déconstruction des faits qu'elle étudie avec l'objectif de montrer comment l'espace est en mesure de les traduire (Chivallon, 2003 ; Di Méo, 2008).

\section{La caméra, un outil de négociation de sa place sur le terrain}

37 Le matériel de tournage permet de négocier une place particulière sur le terrain : la caméra, lorsqu'elle est acceptée par les personnes enquêtées, justifie la présence durable du géographe sur le terrain, elle justifie qu'il se place au cœur de l'action, elle lui permet d'être un observateur actif et non passif. Je rejoins ainsi le point de vue de Marion Ernwein (2015) pour qui le recours à la vidéo dans sa démarche d'observation apparaît comme un moyen de justifier sa présence sur place et de définir sa place au sein des groupes étudiés. La caméra imprime ainsi à tous une temporalité de recherche, puisque sortir sa caméra, c'est se mettre au travail. Elle permet aussi au chercheur de négocier sa place physiquement sur le terrain et de franchir parfois des limites spatiales très intimes. C'est particulièrement vrai lorsqu'on filme une situation de travail. La nécessité de capter un geste implique de se positionner pendant de longues minutes à quelques centimètres de la personne filmée, distance qui serait jugée comme intrusive voir indécente si l'on n'était pas équipée d'une caméra. La rupture de la distance conventionnelle entre filmeur et filmé nécessite souvent un temps préalable d'acceptation du projet de recherche par l'enquêté, d'une reconnaissance et d'une 
appropriation de son utilité, voire de la possibilité d'en tirer un bénéfice personnel. En ce sens, la personne filmée a conscience que si son exposition devant la caméra l'engage personnellement, elle bénéficie en même temps d'une caisse de résonance qui portera beaucoup plus loin l'écho de ses revendications que des gestes et paroles consignés dans des écrits universitaires.

\section{La géographe et la caméra : une autre "production de l'espace »}

La notion de " production de l'espace » a d'abord été formulée dans les années 1960 par les sociologues et les urbanistes, puis reprise par les géographes néo-marxistes dans les décennies suivantes ${ }^{6}$. C'est Henri Lefebvre (1974), partant du constat de la faible théorisation du concept d'espace, qui construit la démonstration du processus de production de l'espace, en insistant sur la combinaison entre " espace perçu », « espace conçu » et « espace vécu ». De façon plus prosaïque, les cinéastes-géographes font très vite le constat sur le terrain que la présence d'une caméra produit un espace particulier, en ce sens où la caméra crée des interactions particulières avec l'espace filmé et les personnages qui y évoluent. Certes, dans tous les cas de pratique de terrain, la présence d'un chercheur dans un espace induit une perturbation, mais cette perturbation est plus ou moins assumée selon que le chercheur se soit présenté ou non, selon qu'il est familier à l'espace étudié, etc. La présence d'une caméra peut ainsi être considérée comme une perturbation parmi d'autres (Buire, 2012).

La présence de la caméra sur un terrain modifie l'organisation de l'espace en imposant un objet physique (l'assemblage caméra, micro et éventuellement trépied) qui peut entraîner une gêne dans les mobilités et les paroles prononcées par les personnes présentes. Elle peut perturber l'action qui se serait déroulée sans la présence de la caméra et entrainer un changement des comportements. Le géographe-cinéaste a le choix de limiter ces perturbations en adoptant des stratégies d'invisibilisation ou au contraire de s'accommoder de ces perturbations, voir de les provoquer. Au cours de nos expériences filmiques de recherche, et particulièrement dans le film Merapi d'un monde à l'autre, avec Florian Geyer nous avons pu alterner les choix. Nous avons parfois tenté de nous faire oublier sur le terrain en nous positionnant dans un coin reculé d'une pièce (dans une arrière-boutique, par exemple, afin de filmer des interactions marchandes) ou nous nous sommes volontairement exposés pour attirer les interactions avec les passants (notamment avec les touristes qui venaient «jouer" autour de notre caméra). Lors du tournage d'Apprentis Chercheurs, j'ai pu observer plusieurs stratégies de la part des étudiants lorsqu'ils étaient en groupe : certains ne semblaient pas être gênés par la présence de la caméra et assumaient leur présence dans le champ, tandis que d'autres sortaient systématiquement du champ par des déplacements de recul discrets et progressifs dès qu'ils se sentaient filmés. S'organisait alors un ballet, entre la filmeuse qui ajustait ses déplacements et ses angles de vue pour tenter de capter le plus d'interactions possibles, les personnes qui acceptaient d'être filmées et celles qui ne voulaient pas, provoquant sans cesse une reconfiguration spatiale du groupe. Dans Entre chiens et loups, alors que nous étions dans une sorte de huis-clos au grand air, la caméra était forcément visible et assumée. Toutefois, son rôle a progressivement évolué au cours du tournage. Dans la première partie du film, la caméra suit les déplacements du berger, elle se place là où il se trouve, elle tente de capter le décor du personnage. Mais au milieu du film, ce n'est plus la caméra qui le suit, mais c'est lui qui vient à la rencontre de la caméra pour venir témoigner des bêtes 
égorgées qu'il vient de trouver : malgré le mauvais temps et sa fatigue apparente, il fait un détour sur le chemin du retour pour venir à la rencontre de ma caméra plutôt que de rentrer directement à la cabane et se mettre au chaud. J'ai monté sciemment cette scène au milieu du film, moment de bascule où le temps tourne à la pluie et où la présence du loup de plus en plus marquante semble faire de la pâture un espace menaçant. À partir de ce moment, le statut de la parole change également : le berger se fait plus confident, moins sûr de lui, évoquant des pensées qui lui sont venues dans la journée. La caméra peut donc tour à tour produire un éloignement ou un rapprochement des corps dans l'espace, mais aussi parfois créer des situations, car elle requiert une performance (Lorimer, 2010).

La caméra a ainsi pour effet de transformer l'espace en une scène d'exposition. En ce sens, la caméra structure l'espace en définissant un lieu du dedans et un lieu du dehors, en distinguant un champ et un hors-champ (Raoulx, 2018). Les personnes filmées peuvent décider de rester sur cette scène ou d'en sortir. Car la présence de la caméra implique que l'espace filmé va être donné à voir et à entendre à un public futur, public qui peut être plus ou moins contrôlé par le chercheur selon la façon dont il va organiser la diffusion de son film, mais qui sera rarement maîtrisé par les protagonistes du film. Là encore, la caméra crée donc un espace négocié entre le chercheur et les filmés. Cette négociation trouvera son issue lors de la projection, dans ce qu'André Gardiès (1993) nomme «l'espace cinématographique » ou l'espace de l'hétérotope "institutionnel » (la salle de projection) dans lequel se trouve immergés ou exposés le spectateur et le filmé (Lévy, 2013). C'est dans ce dernier espace que la personne filmée approuvera ou non le regard porté par le chercheur et validera ou invalidera l'expérience de recherche commune.

\section{Conclusion}

Par l'évocation de mon expérience personnelle de réalisation dans le cadre de mes recherches en géographie, j'ai voulu montrer la manière dont la pratique du cinéma documentaire en géographie peut enrichir la discipline. En me penchant sur mes films mettant en scène des travailleurs et des territoires, j'ai mis en avant la façon dont ces films contribuent à l'enrichissement des savoirs géographiques sur le lien qui unit les travailleurs et leur territoire. J'ai ainsi montré comment les changements environnementaux pouvaient constituer à la fois une menace et une ressource pour les travailleurs évoluant dans des milieux naturels. L'approche incarnée de mes films sur le travail a particulièrement mis en valeur les rapports ambivalents des individus à la nature, rapports qui trouvent leur expression dans l'engagement des corps, mais aussi dans la révélation de liens sentimentaux avec des éléments du territoire. En ce sens, mes films contribuent à l'enrichissement des savoirs en géographie environnementale, mais aussi dans les domaines émergents de la géographie des corps et de la géographie des émotions. J'ai voulu également montrer comment la pratique filmique pousse le géographe à se questionner sur son positionnement vis-à-vis de son objet de recherche et de son terrain d'étude. La caméra engendre en effet un engagement spatial et temporel particulier dont le géographe peut se saisir. Il existe donc bien une spécificité et un intérêt de l'approche documentaire en géographie. Cette approche mériterait d'être institutionnalisée et théorisée comme elle a pu l'être dans le domaine de 
l'anthropologie visuelle (Colleyn, 2012) ou de la sociologie visuelle (Chauvin et Reix, 2015).

\section{BIBLIOGRAPHIE}

Aldhuy J. (2006), « Modes de connaissances, intérêts de connaître et géographie sociale », in Séchet R. et Veschambre (Dir.), Penser et faire la géographie sociale : Contribution à une épistémologie de la géographie sociale, Rennes, PUR, p. 31-46.

Baptiste A. K. (2016), « Can a research film be considered a stand-alone academic publication? An assessment of the film Climate Change, Voices of the Vulnerable : The Fishers' Plight ", Area, vol. $48, \mathrm{n}^{\circ} 4$, p. $463-471$.

Barthe-Deloizy F. (2011), « Le corps peut-il être "un objet" du savoir géographique ? ", Géographie et cultures, $\mathrm{n}^{\circ} 80$, p. 229-247.

Bochet B. et Racine J.-B. (2002), « Connaître et penser la ville : des formes aux affects et aux émotions, explorer ce qu'il nous reste à trouver. Manifeste pour une géographie sensible autant que rigoureuse », Géocarrefour, vol. 77, p. 117-132.

Browaeys X. (1999), « Géographie, image et vidéo. Pour une pratique de l'audiovisuel », L'information géographique, 63-1, p. 25-32.

Buire C. (2011), À travers pratiques citadines et tactiques citoyennes, la production du droit à la ville au Cap (Afrique du Sud), Thèse de doctorat, Université Paris Ouest-Nanterre, 418 p.

Buire C. (2012), « Les arts-de-faire du terrain », Annales de géographie, vol. 5, n 687-688, p. 600-620.

Buob B. (2009), « Filmer, montrer, entendre des savoir-faire. Regards et écoutes croisés dans la médina de Fès », in Fogel F. et I. Rivoal I. (Dir), Ateliers d'anthropologie. Revue éditée par le Laboratoire d'ethnologie et de sociologie comparative, vol. 33.

Calbérac Y. (2010), Terrains de géographes, géographes de terrain. Communauté et imaginaire disciplinaires au miroir des pratiques de terrain des géographes français du XXe siècle, Thèse de doctorat, Université Lumière Lyon 2, 397 p.

Calbérac Y. (2011), « Le terrain des géographes est-il un terrain géographique ? Les terrains de l'épistémologue », Carnets de géographes [En ligne], 2-2011, consulté le 29 juillet 2018. URL :http://carnetsdegeographes.org/carnets_terrain/terrain_02_01_Calberac.php Chauvin P.-M., Reix F. (Dir.) (2015), « Sociologies visuelles », L’Année sociologique, vol. 65, 1.

Chenet M., Simoes L., Laurent Q. (2011), « Pratique et enseignement de l'audiovisuel en géographie », EchoGéo [En ligne], 18, mis en ligne le 05 décembre 2011, consulté le 24 janvier 2019.

Chenet M. (2016), « Le film de recherche, ce bel objet de liberté », in Fontorbes J.-P., Granié A.-M. (Coord.), « Chercheurs de champs », Entrelacs, Hors-série 2, Toulouse, p. 53-59.

Chivallon C. (2003), « Une vision de la géographie sociale et culturelle », Annales de Géographie, $n^{\circ} 634$, p. 646-657. 
Chouraqui F. (2018), Dans la bouche de Fogo, la résilience à l'épreuve des résistances et jeux de pouvoir sur un territoire volcanique, une approche participative et audiovisuelle, Thèse de doctorat, Université Toulouse 2 - Jean Jaurès.

Colleyn J.-P. (2003), Le regard documentaire, Paris, Éditions du Centre Pompidou, 160 p.

Colleyn J.-P. (2012), « Champ et hors champ de l'anthropologie visuelle », L'Homme, 203-204, 457-480.

Collignon B. (2012), Recherches en écriture. Explorations en films documentaires, Mémoire d'Habilitation à Diriger des Recherches, Université Paris-Diderot, Paris.

Davidson J., Bondi L., Smith M. (2005), Emotional geographies, London and New York, Routledge, $272 \mathrm{p}$.

De France C. (1989), Cinéma et anthropologie. Paris, Editions Maison des Sciences de l'Homme.

Di Méo G. (2008), « La géographie culturelle : quelle approche sociale? », Annales de géographie, vol. 660-661, no. 2, p. 47-66.

Di Méo G. (2016), « Une géographie sociale », Cybergeo : European Journal of Geography [En ligne], Les 20 ans de Cybergeo, mis en ligne le 18 août 2016, consulté le 26 juillet 2018. URL : http:// journals.openedition.org/cybergeo/27761

Dwyer C., DaviesS G. (2010), « Qualitative methods III : animating archives, artful interventions and online environments ", Progress in Human Geography, 34(1), p. 88-97.

Ernwein M. (2015), Jardiner la ville néolibérale : la fabrique urbaine de la nature, Thèse de doctorat, Université de Genève, $570 \mathrm{p}$.

Fournand A. (2008), Expériences du corps, expériences de l'espace: une géographie de la maternité et de l'enfantement, Thèse de doctorat, Université de Genève.

Gardiès A. (1993), L'Espace au cinéma, Paris, Klincksieck, 222 p.

Garrett B. L. (2011), “Videographic geographies : Using digital video for geographic research”, Progress in Human Geography, 35(4), p. 521-541.

Garrett B. L. (2013),"Worlds through glass : photography and video as geographic method", in Ward K. (ed.), Researching the City, London, Sage Publications.

Guinard P. (2016), « De la peur et du géographe à Johannesburg (Afrique du Sud) : retour sur des expériences de terrain et propositions pour une géographie des émotion », Géographie et cultures, $\mathrm{n}^{\circ}$ 93-94, p. 277-301.

Guinard P. et Tratnjek P. (2016), « Géographies, géographes et émotions », Carnets de géographes [En ligne], 9 | 2016, mis en ligne le 30 novembre 2016, consulté le 29 juillet 2018. URL : http:// journals.openedition.org/cdg/605

Hancock C., (2011), « Le corps féminin, enjeu géopolitique dans la France postcoloniale », L'Espace politique [en ligne], 13 | 2011-1, mis en ligne le 03 mai 2011, consulté le 29 juillet 2018. URL : http://journals.openedition.org/espacepolitique/1882 ; DOI : 10.4000/espacepolitique.1882

Hémont F., Patrascu M. (2016), « Panorama de méthodologies audiovisuelles en SHS », Revue française des sciences de l'information et de la communication [En ligne], 9, mis en ligne le 01 septembre 2016, consulté le 24 janvier 2019. URL :http://journals.openedition.org/rfsic/2178 ; DOI : $10.4000 /$ rfsic. 2178

Jacobs J. (2015), Visualising the visceral : using film to research the ineffable. Area 48(4), pp. $480-487$. 
Jaurand E. (2015), « La sexualisation des espaces publics dans la subculture gay », Géographie et cultures [En ligne], 95 | 2015, mis en ligne le 12 janvier 2017, consulté le 29 juillet 2018. URL : http://journals.openedition.org/gc/4089

Jewitt C. (2009), The Routledge handbook of multimodal analysis, London and New York, Routledge, $544 \mathrm{p}$.

Kindon S. (2003), "Participatory video in geographic research : a feminist practice of looking ?", Area, 35, 142-153.

Laurier E. (2001), « Why people say where they are during mobile phone call », Environment and Planning D : Society and Space, 19, p. 485-504.

Laurier E. (2005), « Searching for a parking space », Intellectica, 2-3, p. 101-16

Laurier E., Maze R., Lundin J. (2006), « Putting the dog back in the park : animal and human mindin-action. Mind, Culture and Activity, 13, p. 2-24.

Lefebvre H. (1974), La production de l'espace, Paris, Anthropos, 512 p.

Lévy J. (2013), « De l'espace au cinéma », Annales de géographie, n 6, p. 689-711.

Lévy J., Poncet P. et Tricoire E. (2004), La carte, enjeu contemporain, Paris, La documentation française, $63 \mathrm{p}$.

Lorimer J. (2010), « Moving image methodologies for more-than-human geographies », Cultural geographies, $\mathrm{n}^{\circ} 17, \mathrm{p} .237-258$.

Marion J. S., Crowder J. W. (2013), Visual research : a Concise introduction to thinking visually, London, Bloomsbury.

Naville P. (1966). « Instrumentation audio-visuelle et recherche en sociologie », Revue française de sociologie, vol. 7, n² 2, pp. 158-168.

Niney F. (2009), Le documentaire et ses faux-semblants, Paris, Klincksieck, coll. « 50 questions », 207 p.

Pink S. (2011), « Images, senses and applications : engaging visual anthropology », Visual Anthropology, 24, p. 437-54.

Raoulx B. (2009), «Le film documentaire : une méthode pour rendre audiovisible la marginalité (essai sur la démarche géodocumentaire) ", in Bastian S., Bulot T., Burr E. (dir.), Sociolinguistique urbaine et développement durable urbain, Munich, Martin Meidenbauer, pp. 245-269.

Raoulx B. (2018), «L'interdisciplinarité par la création en cinéma documentaire. Retour sur l'expérience du programme FRESH », Revue française des méthodes visuelles [En ligne], 2|2018, mis en ligne le 12 juillet 2018, consulté le 30/01/2019. URL : https://rfmv.fr

Rochefort R. (1961), Travail et travailleurs en Sicile, Paris, Presses Universitaires de France, 363 p.

Rochefort R. (1963a), « Sardes et Siciliens dans les grands ensembles des Charbonnages de Lorraine ", Annales de Géographie, LXXII, n³ 391, p. 272-302.

Rochefort R. (1963b), « Géographie sociale et sciences humaines », Bulletin de l'Association de géographes français, $\mathrm{XL}, \mathrm{n}^{\circ}$ 314, p. 18-32.

Rose G. (2001), Visual methodologies : an introduction to the interpretation of visual materials. Londres, Sage.

Ruby J. (2000), Picturing Culture : Explorations of Film and Anthropology. Chicago, University of Chicago Press. 
Sebag J. (2012), « Sociologie filmique et travail », La nouvelle revue du travail [En ligne], 1 | 2012, mis en ligne le 10 décembre 2012, consulté le 24 janvier 2019. URL :http:// journals.openedition.org/nrt/383 ; DOI : 10.4000/nrt.383

Séchet R. (2012), « De la place des femmes et de leur corps dans la géographie française : souvenirs et expériences personnels ", ESO Travaux et documents, n³3, p. 97-107.

Simpson, P. (2011), “So, as you can see...": some reflections on the utility of video methodologies in the study of embodied practice", Area 43(3), pp. 343-352.

Smith S. J., Anderson K. (2001), “Editorial: Emotional geographies”, Transactions, 26, p. 7-10. Spinney J. (2009), « Cycling the city : movement, meaning and method », Geography Compass, 3, p. 817-35.

Staszak J.-F, Gravari-Barbas M, et Graburn N.H. (2018), « Tout ce que vous voulez savoir sur les sexualités touristiques. Ce qu'on en a su, ce qu'on en sait et ce qu'il reste à en savoir ", Téoros [En ligne], 37, 2 | 2018, mis en ligne le 05 juin 2018, consulté le 08 juin 2018. URL :http:// journals.openedition.org/teoros/3310

Terrenoire J.-P. (1985), « Images et sciences sociales : l'objet et l'outil », Revue française de sociologie, vol. $26, \mathrm{n}^{\circ} 3$, pp. 509-527.

Verne J. (2012), « “Le terrain, c'est moi ?” Reflections on the emergence of the field in translocal research », Annales de géographie, n 687-688, p. 561-582.

Volvey A. (2000), « L'espace vu du corps », in Lévy J. et Lussault M (Dir.), Logiques de l'espace, esprit des lieux, Paris, Belin.

Volvey A. (2003), « Terrain », in Lévy J. et Lussault M. (Dir.), Dictionnaire de la Géographie et de l'Espace des Sociétés, Paris, Belin, p. 904-906.

Volvey A. (2014), « Le corps du chercheur et la question esthétique dans la science géographique ", L'Information géographique, vol. 78, n 1, p. 92-117.

\section{Filmographie}

Apprentis Chercheurs, Marie Chenet, 2013, 34', visible sur https://halshs.archives-ouvertes.fr/ medihal-01178310.

Entre Chiens et loups, Marie Chenet, 2010, 24', visible sur https://vimeo.com/212742493.

Merapi, d'un monde à l'autre, Marie Chenet, Florian Geyer, 2016, 45'.

Traplines in Vancouver, Benoît Raoulx, 2003, 37, visible sur https://www.canal-u.tv/video/ cerimes/traplines_in_vancouver.9476

\section{NOTES}

1. Programme disponible sur https://colloquefilmgeo.wordpress.com/

2. Visible sur https://vimeo.com/212742493.

3. Film visible sur https://halshs.archives-ouvertes.fr/medihal-01178310.

4. DVD à la demande en contactant l'auteur.

5. Notamment, dans l'entretien Serge Daney, itinéraire d'un ciné-fils, monté par Pierre-André Boutang et Dominique Rabourdin diffusé en juin 1992.

6. Pour des éléments de synthèse bibliographique, voir Buire (2011) 


\section{RÉSUMÉS}

La réalisation de films documentaires par des chercheurs est une démarche qui s'est développée en sciences sociales, notamment en anthropologie et en sociologie, mais peu en géographie. En tant que géographe, j'ai réalisé trois films documentaires qui montrent les relations qu'entretiennent les travailleurs avec le milieu naturel, particulièrement en contexte de changements environnementaux. Filmer le travail s'avère particulièrement pertinent pour enrichir les savoirs dans le champ de la géographie environnementale, mais aussi de la géographie culturelle, notamment celui de la géographie des corps et de la géographie des émotions. Au-delà de ces apports thématiques, le recours à la caméra incite le géographecinéaste à s'interroger sur son positionnement de chercheur, à penser sa place sur son terrain d'étude et à envisager son outil comme « producteur » d'un espace particulier.

The production of documentary film by researcher has been developed in social sciences, mainly in anthropology and sociology, slightly less in geography. As a geographer, I have produced three documentary movies that highlight relationships between workers and natural environment, especially in the context of environmental changes. Filming the work is well adapted to enrich knowledge in environmental geography, but contributes also to cultural geography, in the field of body geography and emotional geography. Beyond these themes, the production of research films encourages to clarify researcher's positioning, particularly its own place on the study field and the production of a «new space ».

\section{INDEX}

Mots-clés : travail, cinéma documentaire, géographie filmique

Keywords : work, documentary filmmaking, filmic geography

\section{AUTEUR}

\section{MARIE CHENET}

Maîtresse de Conférences en Géographie à l'Université Paris 1 Panthéon-Sorbonne. Rattachée au Laboratoire de Géographie Physique de Meudon (CNRS-UMR 8591), elle mène des recherches sur les fluctuations glaciaires passées et sur la réponse des sociétés aux changements environnementaux. En parallèle, elle a développé au sein de ses recherches une activité de réalisation de films documentaires. Ses films portent essentiellement sur la façon dont les individus vivent les crises environnementales et plus généralement sur le rapport Homme / Nature. A l'Université, elle enseigne la géomorphologie et forme les étudiants géographes à la réalisation de films documentaires en Licence et en Master. 